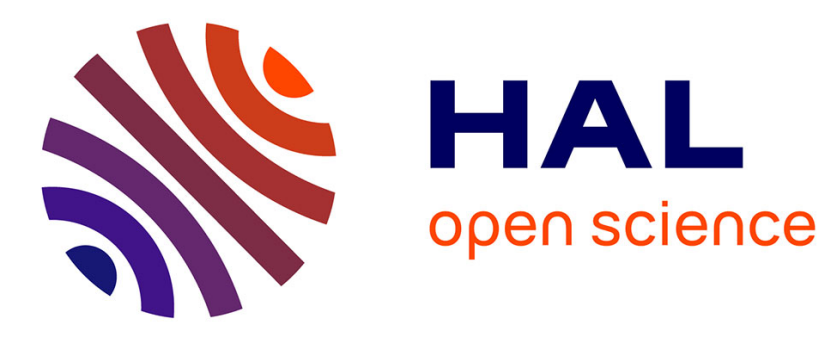

\title{
Energy Efficient Design in MIMO Multi-cell Systems with Time Average QoS Constraints
}

\author{
Subhash Lakshminarayana, Mohamad Assaad, Mérouane Debbah
}

\section{To cite this version:}

Subhash Lakshminarayana, Mohamad Assaad, Mérouane Debbah. Energy Efficient Design in MIMO Multi-cell Systems with Time Average QoS Constraints. 2013 IEEE 14th Workshop on Signal Processing Advances in Wireless Communications (SPAWC), Jun 2013, Darmstadt, Germany. pp.614 618, 10.1109/SPAWC.2013.6612123 . hal-00923436

\section{HAL Id: hal-00923436 \\ https://hal-centralesupelec.archives-ouvertes.fr/hal-00923436}

Submitted on 8 Jan 2014

HAL is a multi-disciplinary open access archive for the deposit and dissemination of scientific research documents, whether they are published or not. The documents may come from teaching and research institutions in France or abroad, or from public or private research centers.
L'archive ouverte pluridisciplinaire HAL, est destinée au dépôt et à la diffusion de documents scientifiques de niveau recherche, publiés ou non, émanant des établissements d'enseignement et de recherche français ou étrangers, des laboratoires publics ou privés. 


\title{
Energy Efficient Design in MIMO Multicell Systems with Time Average QoS Constraints
}

\author{
Subhash Lakshminarayana, Student Member, IEEE, Mohamad Assaad Member, IEEE and Merouane \\ Debbah Senior Member, IEEE
}

\begin{abstract}
In this work, we address the issue of energy efficient design in a MIMO multi-cell network consisting of $N$ cells, $N_{t}$ antennas per BS and $K$ UTs per cell. Under this set up, we address the following question: given certain time average QoS targets for the users, what is the minimum energy expenditure with which they can be met? Time average QoS constraints can lead to greater energy savings as compared to instantaneous QoS constraints since it provides the flexibility to dynamically allocate resources over the fading channel states. We formulate the problem as a stochastic optimization problem whose solution is the design of the downlink beamforming vectors during each time slot. We first characterize the set of time average QoS targets which is achievable by some feasible control policy. We then use the technique of virtual queue to model the time average QoS constraints and convert the problem into a queue stabilization problem while minimizing the time average energy expenditure. We solve this problem using the approach of Lyapunov optimization and characterize its performance. Interestingly, our solution leads to a decentralized design in which the BSs only have to exchange limited side information. Our simulation results show that solving the problem with time average QoS constraints provide greater energy savings as compared to the instantaneous QoS constraints.
\end{abstract}

\section{INTRODUCTION}

Energy efficiency is becoming an important concern in the design of future wireless networks both from environmental and economical point of view. Energy efficient design in cellular networks addresses the concerns of ICT related carbon emissions [1], [2] and leads to a reduction in the costs of running the network due to the reduction in the energy bill [3].

The main focus of this work is energy efficient transmission in MIMO multi-cell networks. Some of the past works focusing on energy efficiency in MIMO systems include [4] (for single user MIMO system), [5], [6] (which consider the effect of circuit power in addition to the transmission power). Recent works focusing on this topic include [7], [8], [9].

In this work, we consider the problem minimizing the time average energy expenditure subject to time average QoS constraints, in a MIMO multi-cell system. The QoS metric we refer to in this work is the difference between the time average useful signal power and the time average interference signal power which is constrained to be greater than the target value. Our motivation to consider the time average QoS constraint comes from the fact that it provides the flexibility

S.Lakshminarayana, M.Assaad are with the Dept. of Telecommunications, SUPELEC, France. S.Lakshminarayana and M. Debbah are also with the Alcatel-Lucent Chair on Flexible Radio, SUPELEC, France. e-mail: \{firstname.lastname\}@supelec.fr. to dynamically allocate resources over the fading channel states as compared to instantaneous QoS constraints. In terms of energy savings, time average QoS constraint can lead to better performance, due to the fact that the transmissions can be delayed until favorable channel conditions are seen, thus minimizing the energy expenditure. The concept has also been exploited in the context of energy-delay trade offs [10], [11].

We formulate our problem as a stochastic optimization problem and propose a solution based on the technique of Lyapunov optimization [12], [13], which provides simple online solutions based only on the current knowledge of the system state (as opposed to traditional approaches such as dynamic programming which suffer from very high complexity and require a-priori knowledge of the statistics of all the random processes in the system). Of particular relevance is the work of [14] which deals with the problem of minimizing the average power expenditure in wireless networks to support the incoming traffic. However, the physical layer model considered is a simple conflict graph based model in which if two transmitters are within the interference range of each other, only one of them can transmit. Therefore, the rate achieved depends only on the power allocated to the link which is scheduled (and hence the signal to noise ratio (SNR) of the link). However, advances in physical layer techniques like multiple antenna technologies allow multiple users to coexist over the same resource block. Therefore, in this work, we consider the problem of energy efficient transmissions in a MIMO multi-cell scenario.

Our contributions in this work are as follows.

- We first formulate the feasible $Q o S$ region, i.e. the set of target QoS constraints that is achievable by some control policy.

- We model the time average constraint as a virtual queue and transform the problem into a queue stabilization problem while minimizing the average energy expenditure. We then use the technique of Lyapunov optimization [12], [13] to formulate a dynamic control strategy that satisfies the time average QoS targets. Our algorithm leads to a decentralized design in which the BSs can formulate the beamforming vectors using only the local CSI. The BSs would only have to exchange virtual queue-length information among themselves.

- We provide the performance bounds for our decentralized algorithm and show that its performance in terms of the energy expenditure can be made arbitrarily close to the energy expenditure of the optimal policy. 


\section{SySTEM MODEL}

We consider a MIMO multi-cell scenario consisting $N$ cells and $K$ UTs per cell. The UTs in each cell are served by their respective BSs which are equipped with $N_{t}$ antennas. The UTs have a single antenna each. The notation $\mathrm{UT}_{i, j}$ denotes the $j$ th UT present in the $i$-th cell. The BS of each cell serves only the UTs present in its cell. We consider a discrete-time blockfading channel model where the channel remains constant for a given coherence interval and then changes independently from one block to the other. We index the time slots by $t$. We denote the channel vector from the $\mathrm{BS}_{i}$ to the $\mathrm{UT}_{j, k}$ during the time slot $t$ by $\mathbf{h}_{i, j, k}[t] \in \mathbb{C}^{N_{t}}$. We define the channel matrix $\mathbf{H}[t]$ given $\mathbf{H}_{i, j}[t]=\left[\mathbf{h}_{i, j, 1}[t], \ldots, \mathbf{h}_{i, j, K}[t]\right]$; $\mathbf{H}_{i}[t]=\left[\mathbf{H}_{i, 1}[t], \ldots, \mathbf{H}_{i, N}[t]\right]$ and $\mathbf{H}[t]=\left[\mathbf{H}_{i}[t], \ldots, \mathbf{H}_{N}[t]\right]$. The channel process $\{\mathbf{H}[t], t=0,1,2, \ldots\}$ is assumed to be an independent and identically distributed (i.i.d) discrete time stationary ergodic random process which takes values from the finite state space $\left\{\mathcal{H}_{1}, \ldots, \mathcal{H}_{L}\right\}$. We denote the distribution $\operatorname{Pr}\left(\mathbf{H}[t]=\mathcal{H}_{l}\right)=\pi_{l}, \quad l=1, \ldots, L$ where $\sum_{l} \pi_{l}=1$. Let us denote the beamforming vector corresponding to $\mathrm{UT}_{i, j}$ during slot $t$ by $\mathbf{w}_{i, j}[t] \in \mathbb{C}^{N_{t}}$. The signal received by $\mathrm{UT}_{i, j}$ during time $t$ is given by

$$
\begin{aligned}
y_{i, j}[t]=\mathbf{h}_{i, i, j}^{H}[t] \mathbf{w}_{i, j}[t] x_{i, j}[t]+ & \sum_{\substack{(n, k) \\
\neq(i, j)}} \mathbf{h}_{n, i, j}^{H}[t] \mathbf{w}_{n, k}[t] x_{n, k}[t] \\
& +z_{i, j}[t]
\end{aligned}
$$

where $x_{i, j}[t] \in \mathbb{C}$ represents the information signal for the $\mathrm{UT}_{i, j}$ during the time slot $t$ and $z_{i, j} \sim \mathcal{C N}\left(0, N_{0}\right)$ is the corresponding additive white Gaussian complex noise. The QoS metric which we denote by $\gamma_{i, j}[t]$ is

$$
\gamma_{i, j}[t]=\left|\mathbf{w}_{i, j}^{H}[t] \mathbf{h}_{i, i, j}[t]\right|^{2}-\sum_{\substack{(n, k) \\ \neq(i, j)}}\left|\mathbf{w}_{n, k}^{H}[t] \mathbf{h}_{n, i, j}[t]\right|^{2} .
$$

\section{Comment on the QoS metric:}

The QoS metric chosen in this work represents the difference between time average useful signal power and the time average interference signal power. In addition to multi-cell networks, the QoS metric in our work can be applied to cellular networks with cognitive network underlay. In this context, one can view the QoS metric in similar spirit with metrics such as interference temperature control [15], [16], [17], [18] (in which the interference, peak interference power (PIP) or average interference power (AIP) is constrained to below a certain threshold). Our QoS metric is more general in the sense that we constraint the interference signal to be below the useful signal power upto a threshold level. The target QoS values can be adjusted to different values depending on the tier to which the users belong, e.g. the primary users can have higher QoS target (since the primary users are licensed users and hence demand greater QoS guarantees) and the cognitive users can have a lower QoS target. In this work, we consider the time average QoS constraints as it can provide more flexibility as compared to instantaneous constraints in terms of dynamically allocating resources over fading channel states, thus leading to better system performance. It has been shown in some works in the past that time average QoS constraints lead to better system performance as compared to the instantaneous (peak) constraints [17], [18].

The transmission power by each BS $P_{i}[t]$ depends on the beamforming vector during the time slot $t$ which can be given as $P_{i}[t]=\sum_{j=1}^{K} \mathbf{w}_{i, j}^{H}[t] \mathbf{w}_{i, j}[t], \quad i=1, \ldots, N$. The optimization problem to minimize the average energy expenditure subject to time average QoS constraint can be formulated as

$$
\begin{array}{ll}
\min & \lim _{T \rightarrow \infty} \frac{1}{T} \sum_{t=0}^{T-1} \mathbb{E}\left[\sum_{i=1}^{N} P_{i}[t]\right] \\
\text { s.t. } & \lim _{T \rightarrow \infty} \frac{1}{T} \sum_{t=0}^{T-1} \mathbb{E}\left[\gamma_{i, j}[t]\right] \geq \lambda_{i, j}, \quad \forall i, j \\
& \sum_{j=1}^{K} \mathbf{w}_{i, j}^{H}[t] \mathbf{w}_{i, j}[t] \leq P_{\text {peak }} \quad \forall i, t
\end{array}
$$

where $P_{\text {peak }}$ is the peak power at which the BSs can transmit.

The optimization problem in (3) is a stochastic optimization problem. The control action to be taken during each time slot is the formulation of the downlink beamforming vectors $\left(\mathbf{w}_{i, j}[t] \forall i, j\right)$ during every time slot $t$. In particular, we search for the sequence of control actions which result in minimum time average energy expenditure while satisfying the time average QoS constraints.

We will henceforth denote the time average quantities by $\bar{x}=\lim _{T \rightarrow \infty} \frac{1}{T} \sum_{t=0}^{T-1} \mathbb{E}[x[t]]$.

Let $P_{\text {inf }}$ be the infimum time average power $\bar{P}$ incurred while achieving the time average QoS targets over all possible sequence of control actions. In this work, we use the technique of Lyapunov optimization to provide a simple online solution to the stochastic optimization problem.

\section{AchiEvable QoS REgION}

Before we solve (3), we will first characterize the set of all the time average QoS targets which can be satisfied by some control policy. Problem (3) is solvable only if the time average QoS constraints lie within this region. Let us define the matrices $\mathbf{W}_{i}=\left[\mathbf{w}_{i, 1}, \ldots, \mathbf{w}_{i, K}\right] \in \mathbb{C}^{N_{t} \times K}$ and $\mathbf{W}=$ $\left[\mathbf{W}_{1}, \ldots, \mathbf{W}_{N}\right] \in \mathbb{C}^{N_{t} \times N K}$. We denote the collection of all feasible beamforming vectors $\mathbf{W}$ by the set $\mathcal{W}$ which can be characterized as follows,

$$
\begin{aligned}
& \mathcal{W}=\left\{\mathbf{W}: \mathbf{w}_{i, j} \in \mathbb{C}^{N_{t}} \forall i, j\right. \\
& \text { s.t. } \left.\sum_{j} \mathbf{w}_{i, j}^{H} \mathbf{w}_{i, j} \leq P_{\max }, \forall i\right\} .
\end{aligned}
$$

The QoS associated with a particular beamforming policy $\mathbf{W}$ when the channel is in state $l$ is

$$
\gamma_{i, j}(l, \mathbf{W})=\left|\mathbf{h}_{i, i, j}^{H} \mathbf{w}_{i, j}\right|^{2}-\sum_{\substack{(n, k) \\ \neq(i, j)}}\left|\mathbf{h}_{n, i, j}^{H} \mathbf{w}_{n, k}\right|^{2} .
$$

We also define the vectors $\gamma_{i}(l, \mathbf{W})=\left[\gamma_{i, 1}, \ldots, \gamma_{i, K}\right] \in \mathbb{R}^{K}$ and $\gamma(l, \mathbf{W})=\left[\gamma_{1}, \ldots, \gamma_{N}\right] \in \mathbb{R}^{N K}$. The achievable $Q o S$ region is then given by

$$
\boldsymbol{\Gamma}=\left\{\boldsymbol{\lambda} \in \mathbb{R}^{N K}: \boldsymbol{\lambda} \leq \sum_{l} \pi_{l} \mathcal{C H}(\gamma(l, \mathbf{W}) \mid \mathbf{W} \in \mathcal{W})\right\}
$$


where $\mathcal{C H}(\mathbf{A})$ represents the convex hull of the set $\mathbf{A}$.

The basic idea here is that by time sharing between different beamforming vectors in the set $\mathcal{W}$, any QoS constraint inside the region defined by the convex hull should be achievable. The achievable QoS region of the form (11) is similar to the characterization of the stability region in the case of scheduling algorithms in wireless networks(e.g. see Theorem 3.6, [12]). The result state more formally is as follows: for any target QoS lying inside the stability region $(\lambda \in \Gamma)$, there exists a stationary randomized policy that can achieve the target QoS [19], Theorem 4.5.

\section{ENERGY EFFICIENT DECENTRALIZED BEAMFORMING DESIGN}

Before we solve (3), we will first characterize the set of all the time average QoS targets which can be satisfied by some control policy. Problem (3) is solvable only if the time average QoS constraints lie within this region. Let us define the matrices $\mathbf{W}_{i}=\left[\mathbf{w}_{i, 1}, \ldots, \mathbf{w}_{i, K}\right] \in \mathbb{C}^{N_{t} \times K}$ and $\mathbf{W}=$ $\left[\mathbf{W}_{1}, \ldots, \mathbf{W}_{N}\right] \in \mathbb{C}^{N_{t} \times N K}$. We denote the collection of all feasible beamforming vectors $\mathbf{W}$ by the set $\mathcal{W}$ which can be characterized as follows,

$$
\begin{aligned}
& \mathcal{W}=\left\{\mathbf{W}: \mathbf{w}_{i, j} \in \mathbb{C}^{N_{t}} \forall i, j\right. \\
& \text { s.t. } \left.\sum_{j} \mathbf{w}_{i, j}^{H} \mathbf{w}_{i, j} \leq P_{\max }, \forall i\right\} .
\end{aligned}
$$

The QoS associated with a particular beamforming policy $\mathbf{W}$ when the channel is in state $l$ is

$$
\gamma_{i, j}(l, \mathbf{W})=\left|\mathbf{h}_{i, i, j}^{H} \mathbf{w}_{i, j}\right|^{2}-\sum_{\substack{(n, k) \\ \neq(i, j)}}\left|\mathbf{h}_{n, i, j}^{H} \mathbf{w}_{n, k}\right|^{2} .
$$

We also define the vectors $\gamma_{i}(l, \mathbf{W})=\left[\gamma_{i, 1}, \ldots, \gamma_{i, K}\right] \in \mathbb{R}^{K}$ and $\gamma(l, \mathbf{W})=\left[\gamma_{1}, \ldots, \gamma_{N}\right] \in \mathbb{R}^{N K}$. The achievable $Q o S$ region is then given by

$$
\boldsymbol{\Gamma}=\left\{\boldsymbol{\lambda} \in \mathbb{R}^{N K}: \boldsymbol{\lambda} \leq \sum_{l} \pi_{l} \mathcal{C H}(\gamma(l, \mathbf{W}) \mid \mathbf{W} \in \mathcal{W})\right\}
$$

where $\mathcal{C H}(\mathbf{A})$ represents the convex hull of the set $\mathbf{A}$.

The basic idea here is that by time sharing between different beamforming vectors in the set $\mathcal{W}$, any QoS constraint inside the region defined by the convex hull should be achievable. The achievable QoS region of the form (11) is similar to the characterization of the stability region in the case of scheduling algorithms in wireless networks(e.g. see Theorem 3.6, [12]). The result state more formally is as follows: for any target QoS lying inside the stability region $(\boldsymbol{\lambda} \in \boldsymbol{\Gamma})$, there exists a stationary randomized policy that can achieve the target QoS [19], Theorem 4.5.

\section{ENERGy EFFICIENT DECENTRALIZED BEAMFORMING DESIGN}

In this work, in order to model the time average QoS constraint, we use the concept of virtual queиe [19]. The virtual queue associated with the time average constraint $\bar{\gamma}_{i, j} \geq \lambda_{i, j}$ evolves in the following manner,

$$
Q_{i, j}[t+1]=\max \left(Q_{i, j}[t]-\mu_{i, j}[t], 0\right)+A_{i, j}[t]
$$

where $A_{i, j}[t]=\sum_{\substack{(n, k) \\ \neq(i, j)}}\left|\mathbf{w}_{n, k}^{H}[t] \mathbf{h}_{n, i, j}[t]\right|^{2}+\lambda_{i, j}$ denotes the arrival process and $\mu_{i, j}[t]=\left|\mathbf{w}_{i, j}^{H}[t] \mathbf{h}_{i, i, j}[t]\right|^{2}$ denotes the departure process The notion of strong stability of the virtual queue is given as $\sum_{i, j} \bar{Q}_{i, j}[t]<\infty$. Ensuring the strong stability of the virtual queue implies that the lim of time average of the arrival process is less than or equal to the service process [19] 1 , i.e.

$$
\left.\bar{A}_{i, j}[t]-\bar{\mu}_{i, j}[t]\right] \leq 0 \quad \forall i, j .
$$

In other words, the constraint (4) is satisfied. Thus, we reformulate the original problem into a problem of stabilizing the virtual queue while minimizing the time average energy expenditure.

In order to solve this, we use the technique of Lyapunov optimization [12] which allows us to consider the joint problem of stabilizing the queue and performance optimization.

To this end, we define the quadratic Lyapunov function $V$ : $\mathbb{R}^{N} \rightarrow \mathbb{R}$ as: $V(\mathbf{Q}[t])=\frac{1}{2} \sum_{i, j}\left(Q_{i, j}[t]\right)^{2}$. The Lyapunov function is a scalar measure of the aggregate queue-lengths in the system. We define the one-step conditional Lyapunov drift as

$$
\Delta(\mathbf{Q}[t])=\mathbb{E}[V(\mathbf{Q}[t+1]))-V(\mathbf{Q}[t]) \mid \mathbf{Q}[t]]
$$

where the expectation is with respect to the random channel states and the (possibly random) control actions made in reaction to these channel states.

We will now examine the Lyapunov drift corresponding to the evolution of the virtual queue $Q_{i, j}$.

Proposition 1. For the virtual queue which evolves according to (12), the Lyapunov drift follows the following condition.

$$
\begin{gathered}
\Delta(\mathbf{Q}[t])+V \mathbb{E}\left[\sum_{i, j} \mathbf{w}_{i, j}^{H}[t] \mathbf{w}_{i, j}[t] \mid \mathbf{Q}[t]\right] \leq C_{1} \\
+\sum_{i, j} Q_{i, j}[t] \lambda_{i, j}-\sum_{i, j} \mathbb{E}\left[Q _ { i , j } [ t ] \left(\left|\mathbf{w}_{i, j}^{H}[t] \mathbf{h}_{i, i, j}[t]\right|^{2}\right.\right. \\
\left.\quad-\sum_{\substack{(n, k) \\
\neq(i, j)}}\left|\mathbf{w}_{n, k}^{H}[t] \mathbf{h}_{n, i, j}[t]\right|^{2}\right) \\
\left.-V \mathbf{w}_{i, j}^{H}[t] \mathbf{w}_{i, j}[t] \mid \mathbf{Q}[t]\right] \quad \forall t
\end{gathered}
$$

where $C_{1}=\frac{1}{2} \sum_{i, j} \mathbb{E}\left[\left(A_{i, j}^{\max }[t]\right)^{2}+\left(\mu_{i, j}^{\max }[t]\right)^{2}\right]<\infty$ and

$$
\begin{aligned}
& A_{\max }[t]=N P_{\text {peak }} \max _{n}\left|\mathbf{h}_{n, i, j}[t]\right|^{2}+\lambda_{i, j} \\
& \mu_{\max }[t]=P_{\text {peak }}\left|\mathbf{h}_{i, i, j}[t]\right|^{2} .
\end{aligned}
$$

The proof follows from the steps in Appendix, part I. According to the theory of Laypunov optimization, a good method to choose the beamforming vector is to minimize the bound obtained in (15). This implies that the beamforming vector should be chosen in the following manner:

$$
\begin{array}{r}
\mathbf{w}_{i, j}[t] \quad \in \arg \max _{\mathbf{w} \in \mathcal{W}} \sum_{i, j} \mathbb{E}_{\mathbf{H}}\left[Q_{i, j}[t]\left|\mathbf{w}_{i, j}^{H} \mathbf{h}_{i, i, j}[t]\right|^{2}\right. \\
\left.-Q_{i, j}[t] \sum_{\substack{(n, k) \\
\neq(i, j)}}\left|\mathbf{w}_{n, k}^{H} \mathbf{h}_{n, i, j}[t]\right|^{2}-V \mathbf{w}_{i, j}^{H} \mathbf{w}_{i, j} \mid \mathbf{Q}(t)\right]
\end{array}
$$

\footnotetext{
${ }^{1}$ In general mean rate stability is sufficient to ensure this condition. Here we use a stronger notion of stability.
} 
where $\mathbb{E}_{\mathbf{H}}$ indicates that the expectation is with respect to the random channel realization. We will assume that the BSs have the perfect knowledge of CSI of all its downlink channels $\left(\mathbf{h}_{i, n, k} \forall n, k\right)$ and propose a method to solve (18). Interestingly, our problem approach also leads to a decentralized solution in the multi-cell scenario. Further, we will theoretically examine some properties of this algorithm and analyze its performance.

With the perfect knowledge of CSI, the optimization problem (18) reduces to greedily minimizing the term inside the expectation $(\mathbb{E}[f(Y) \mid Y]=f(Y)$ ). Therefore, we remove the expectation and solve the following optimization problem (we drop the time index $t$ ),

$$
\begin{aligned}
\max _{\mathbf{w} \in \mathbb{C}^{N_{t}}} & \sum_{i, j}\left[Q_{i, j}\left|\mathbf{w}_{i, j}^{H} \mathbf{h}_{i, i, j}\right|^{2}\right. \\
& \left.-Q_{i, j} \sum_{\substack{(n, k) \\
\neq(i, j)}}\left|\mathbf{w}_{n, k}^{H} \mathbf{h}_{n, i, j}\right|^{2}-V \mathbf{w}_{i, j}^{H} \mathbf{w}_{i, j}\right] \\
\text { s.t. } & \sum_{j} \mathbf{w}_{i, j}^{H} \mathbf{w}_{i, j} \leq P_{\text {peak }} \quad \forall i .
\end{aligned}
$$

The objective function of the optimization problem in (19) can be rearranged and written as,

$$
\begin{array}{ll}
\max _{\mathbf{w}} & \sum_{i, j} \mathbf{w}_{i, j}^{H} \mathbf{A}_{i, j} \mathbf{w}_{i, j} \\
\text { s.t. } & \sum_{j} \mathbf{w}_{i, j}^{H} \mathbf{w}_{i, j} \leq P_{\text {peak }} \quad \forall i
\end{array}
$$

where the matrix $\mathbf{A}_{i, j}=Q_{i, j} \mathbf{H}_{i, i, j}-\sum_{\substack{(n, k) \\ \neq(i, j)}} Q_{n, k} \mathbf{H}_{i, n, k}-V \mathbf{I}$ and $\mathbf{H}_{i, n, k}=\mathbf{h}_{i, n, k} \mathbf{h}_{i, n, k}^{H}$. Note that the optimization problem in (20) is in separable form, each BS $i$ can solve the optimization problem given by

$$
\begin{array}{ll}
\max _{\mathbf{w}} & \sum_{j} \mathbf{w}_{i, j}^{H} \mathbf{A}_{i, j} \mathbf{w}_{i, j} \\
\text { s.t. } & \sum_{j} \mathbf{w}_{i, j}^{H} \mathbf{w}_{i, j} \leq P_{\text {peak }} .
\end{array}
$$

Denoting $\mathbf{W}_{i, j}=\mathbf{w}_{i, j}^{H} \mathbf{w}_{i, j}$, (21) can now be rewritten in matrix form as

$$
\begin{array}{ll}
\max _{\mathbf{W}} & \sum_{j} \operatorname{tr}\left(\mathbf{A}_{i, j} \mathbf{W}_{i, j}\right) \\
\text { s.t. } & \sum_{j} \operatorname{tr}\left(\mathbf{W}_{i, j}\right) \leq P_{\text {peak }} \\
& \operatorname{rank}\left(\mathbf{W}_{i, j}\right)=1 \quad \forall j .
\end{array}
$$

Relaxing the rank constraint on the $\mathbf{W}_{i, j}$ matrices in (22) leads to a semi-definite programming (SDP) problem which can be solved optimally using the optimization package SEDUMI [20]. Also, from the result of [21], it is easy to see that the solution obtained form the relaxed problem is always rank 1. SDP problems can be efficiently solved using interior point methods, at a complexity cost of at most $O\left(N+N_{t}^{2}\right)^{3.5}$. Later on in this section, we will provide an algorithm to solve the optimization problem 21 with a lower complexity.

Observe that in order to formulate the matrix $\mathbf{A}_{i, j}$, the $\mathrm{BSs}$ only require the local CSI $\left(\mathbf{h}_{i, n, k} \forall n, k\right)$. The BSs would only have to exchange the queue-lengths among themselves.
Therefore, our formulation naturally leads to a decentralized solution.

We will hereby address the algorithm corresponding to solving (21) as the decentralized beamforming (DBF) algorithm. We will also denote the solution corresponding to (21) by the superscript "opt". We will now provide some theoretical analysis into the performance of the DBF algorithm.

Proposition 2. Under the DBF strategy, during every time slot $t$, it is optimal for BS to serve at most one UT per cell. Further, during a given time slot $t$, if the BS is serving a UT, then it is optimal to serve it at its peak power.

Proof: Proposition 2 can be argued as follows. Recall the optimization problem in (20). Let us decompose the beamforming vector into power allocation and direction vector as follows. $\left\|\mathbf{w}_{i, j}\right\|^{2}=P_{i, j}$ and $\mathbf{d}_{i, j}=\frac{\mathbf{w}_{i, j}}{\left\|\mathbf{w}_{i, j}\right\|}$. Optimization problem in (20) can now be written as

$$
\begin{array}{cl}
\max _{P_{i, j},\left\|\mathbf{d}_{i, j}\right\|=1} & \sum_{j} P_{i, j} \mathbf{d}_{i, j}^{H} \mathbf{A}_{i, j} \mathbf{d}_{i, j} \\
\text { s.t. } & \sum_{j} P_{i, j} \leq P_{\text {peak }}
\end{array}
$$

Noting that $\max _{\left\|\mathbf{d}_{i, j}\right\|=1} \mathbf{d}_{i, j}^{H} \mathbf{A}_{i, j} \mathbf{d}_{i, j}=\lambda^{\max }\left(\mathbf{A}_{i, j}\right)$ (Rayleigh-Ritz theorem), where $\lambda^{\max }\left(\mathbf{A}_{i, j}\right)$ is the maximum eigen value of the matrix $\mathbf{A}_{i, j}$, it can be verified that the optimization problem in (23) can be solved as

$$
\max _{P_{i, j} \leq P_{\text {peak }}} \sum_{j} P_{i, j} \lambda^{\max }\left(\mathbf{A}_{i, j}\right)
$$

Let us define $j^{*}=\arg \max _{j} \lambda^{\max }\left(\mathbf{A}_{i, j}\right)$. Therefore from (24), it is straightforward to see that,

$$
P_{i, j}^{\mathrm{opt}}= \begin{cases}P_{\text {peak }} & \text { if } j=j^{*} \text { and } \lambda^{\max }\left(\mathbf{A}_{i, j^{*}}\right)>0 \\ 0 & \text { else. }\end{cases}
$$

Proposition 2 implies that at most one UT can be active per cell during each time slot. During a given time slot $t$, in every cell, there can be either one active UT in the cell which case the BS transmits at its peak power $\left(P_{i}^{\mathrm{opt}}[t]=P_{\text {peak }}\right)$ or there are no active UTs in the cell and hence the BS does not transmit $\left(P_{i}^{\mathrm{opt}}[t]=0\right)$. Also, we can conclude that

$$
\sum_{j} \operatorname{tr}\left(\mathbf{A}_{i, j}[t] \mathbf{W}_{i, j}^{\mathrm{opt}}[t]\right)=P_{\text {peak }} \lambda^{\max }\left(\mathbf{A}_{i, j^{*}}\right) \mathbf{1}_{\lambda^{\max }\left(\mathbf{A}_{i, j^{*}}\right)>0} .
$$

Proposition 2 also provides us an easier method to solve the optimization problem (19). It can be seen that the optimal beamforming vector during each time slot is the eigen vector corresponding to the maximum eigen value $\left(\lambda^{\max }\left(\mathbf{A}_{i, j^{*}}\right)\right.$.) Therefore,

$$
\mathbf{w}_{i, j^{*}}^{\text {opt }}=\left(P_{\text {peak }} \lambda^{\max }\left(\mathbf{A}_{i, j^{*}}\right) \mathbf{1}_{\lambda^{\max }\left(\mathbf{A}_{i, j^{*}}\right)>0}\right) \mathbf{x}_{\lambda^{\max }\left(\mathbf{A}_{i, j^{*}}\right)}
$$

where $\mathbf{x}_{\lambda^{\max }\left(\mathbf{A}_{i, j^{*}}\right)}$ is the eigen vector corresponding to the maximum eigen value of matrix $\mathbf{A}_{i, j^{*}}$ and

$$
\mathbf{w}_{i, j}^{\mathrm{opt}}=\mathbf{0} \quad j^{*} \neq j .
$$


At this stage, we would like to point out that the complexity of eigenvalue decomposition function is $O\left(N_{t}^{3}\right)$ which is lower than the complexity of the SDP based solution. Therefore, 26 offers an easier method to calculate the optimal beamforming vectors with lesser computational complexity.

\section{Intuition:}

Taking a closer look at the optimization problem (22), it can be seen that each $\mathrm{UT}_{i, j}$ has a metric associated with it given by,

$$
\begin{aligned}
\operatorname{tr}\left(\mathbf{A}_{i, j} \mathbf{W}_{i, j}\right) & =\operatorname{tr}(Q_{i, j} \underbrace{\mathbf{H}_{i, i, j} \mathbf{W}_{i, j}}_{\text {Useful signal }} \\
& -\sum_{\substack{(n, k) \\
\neq(i, j)}} Q_{n, k} \underbrace{\mathbf{H}_{i, n, k} \mathbf{W}_{i, j}}_{\text {Interference signal to other users }}-V \mathbf{W}_{i, j}) .
\end{aligned}
$$

The metric corresponds to the difference between weighted sum of the useful signal (to the $\mathrm{UT}_{i, j}$ ) and the weighted sum of interference caused to the other users in the system $\left(\mathrm{UT}_{(n, k) \neq(i, j)}\right)$. The weights are the corresponding queuelength values which indicate how urgently the UT needs to be served. Therefore, intuitively, each BS schedules the UT in its cell which has the highest value of this metric $\lambda^{\max }\left(\mathbf{A}_{i, j^{*}}\right)$. Additionally, the transmission direction corresponds to the eigen vector corresponding to the $\left(\lambda^{\max }\left(\mathbf{A}_{i, j^{*}}\right)\right)$. The parameter $V$ represents how aggressively the BS decides to transmit. Higher value of $V$ implies less aggressive transmission and greater energy savings.

We will now proceed to provide performance bounds of the DBF algorithm in terms of average power consumption and average backlog.

Proposition 3. For any target SINR lying strictly inside the feasible QoS region, the DBF algorithm yields the following performance bounds. The virtual queue is strongly stable and for any $V \geq 0$, the time average queue-length satisfies $\sum_{i, j} \bar{Q}_{i, j}^{\text {opt }}[t] \leq \frac{C_{1}+V N K P_{\text {peak }}}{\epsilon}$ and the time average energy expenditure yields, $\sum_{i=1}^{N} \bar{P}_{i}^{\text {opt }}[t] \leq P_{\mathrm{inf}}+\frac{C_{1}}{V}$.

The proof follows from the steps in Appendix, part II. The bound of Proposition 3 implies that the time average energy expended by the DBF algorithm can be made arbitrarily close to the minimum average power (over all possible sequence on control actions) by increasing the value of $V$ to arbitrarily an high value. This comes at the expense of increasing the average queue-length of the virtual queue. Intuitively, a high value of the average queue-length implies that the number of time slots required to satisfy the time average constraints is higher (analogous to the concept of delay in real queues).

\section{NUMERICAL RESUlTS}

In this section, we present some numerical results to demonstrate the performance of the DBF algorithm. We consider a system consisting of 2 cells with each cell having 2 UTs each. Each BS has 5 antennas and $P_{\text {peak }}=10 \mathrm{~dB}$ per BS. We consider a distance dependent path loss model, the path loss factor from from $\mathrm{BS}_{i}$ to $\mathrm{UT}_{j, k}$ is given as $\sigma_{i, j, k}=d_{i, j, k}^{-\beta}$ where $d_{i, j, k}$ is the distance between $\mathrm{BS}_{i}$ to $\mathrm{UT}_{j, k}$, normalized to the maximum distance within a cell, and $\beta$ is the path loss exponent (in the range from 2 to 5 dependent on the radio environment).

We plot the time average energy expenditure per BS versus the target QoS for two cases. In the first case, we solve the problem of minimizing the instantaneous energy expenditure subject to instantaneous QoS constraints ( $\min _{\mathbf{w}} \sum_{i=1}^{N} \sum_{j=1}^{K} \mathbf{w}_{i, j}^{H}[t] \mathbf{w}_{i, j}[t]$ s.t. $\left.\gamma_{i, j}[t] \geq \lambda_{i, j} \forall t\right)$. We repeat this for 1000 time slots. In the second scenario, we solve the problem of minimizing the time average energy expenditure subject to time average QoS constraints $\left(\bar{\gamma}_{i, j} \geq \lambda_{i, j}\right)$. We plot the result in Figure 1 It can be seen that for the case with time average constraints, the energy expenditure is lower. In particular, for a target QoS of $10 \mathrm{~dB}$, energy minimization with time average QoS constraints under the Lyapunov optimization based approach provides upto $4 \mathrm{~dB}$ reduction in the energy expenditure as compared to the case with instantaneous QoS constraints (for $V=800$.) This is in accordance with our intuition that the time average QoS constraint provides greater flexibility in allocating resources over channel fading states.

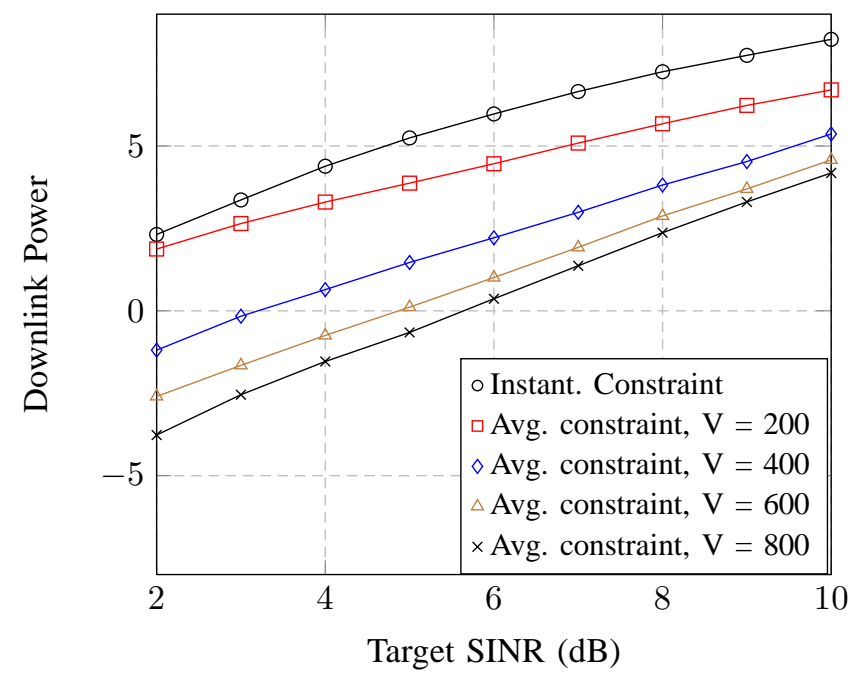

Fig. 1. Average energy expenditure Vs target QoS for a two cell scenario, each cell consisting of two UTs, $N_{t}=5, P_{\text {peak }}=10 \mathrm{~dB}$.

\section{CONCLUSION}

In this work, we handled the problem of minimizing the time average energy expenditure subject to satisfying time average QoS constraints in a MIMO multi-cell scenario. Using the technique of Lyapunov optimization, we proposed a decentralized online beamforming design algorithm whose performance in terms of the time average expenditure can be made arbitrarily close to the optimal. Our results show that time average QoS constraints can lead to better savings in terms of energy expenditure as compared to solving the problem with instantaneous constraints. 


\section{APPENDIX : PERFORMANCE BOUNDS}

Part 1: Proof of Proposition 1

From (12), we can write the following.

$$
\begin{aligned}
Q_{i, j}^{2}[t+1] & \leq\left(Q_{i, j}[t]-\mu_{i, j}[t]\right)^{2}+A_{i, j}^{2}[t] \\
& +2 A_{i, j}[t] \max \left(0, Q_{i, j}[t]-\mu_{i, j}[t]\right) \\
& \leq Q_{i, j}^{2}[t]+\mu_{i, j}^{2}[t]+A_{i, j}^{2}[t] \\
& -2 Q_{i, j}[t]\left(\mu_{i, j}[t]-A_{i, j}[t]\right) .
\end{aligned}
$$

Summing with respect to $i, j$ and taking the conditional expectation $\mathbb{E}[. \mid \mathbf{Q}[t]]$, we have,

$$
\begin{aligned}
\Delta(\mathbf{Q}[t]) & \leq \sum_{i, j} \mathbb{E}\left[\mu_{i, j}^{2}[t]+A_{i, j}^{2}[t] \mid \mathbf{Q}[t]\right] \\
& -\sum_{i, j} Q_{i, j}[t] \mathbb{E}\left[\mu_{i, j}[t]-A_{i, j}[t] \mid \mathbf{Q}[t]\right]
\end{aligned}
$$

Now, we can provide the following bounds,

$$
\begin{aligned}
A_{i, j}[t] & =\sum_{\substack{(n, k) \\
\neq(i, j)}}\left|\mathbf{w}_{n, k}^{H}[t] \mathbf{h}_{n, i, j}[t]\right|^{2}+\lambda_{i, j} \\
\leq & P_{\text {peak }} \sum_{n}\left|\mathbf{h}_{n, i, j}[t]\right|^{2}+\lambda_{i, j} \\
\leq & N P_{\text {peak }} \max _{n}\left|\mathbf{h}_{n, i, j}[t]\right|^{2}+\lambda_{i, j} \triangleq A_{i, j}^{\max }[t]
\end{aligned}
$$

and

$$
\begin{aligned}
\mu_{i, j}[t] & =\left|\mathbf{w}_{i, j}^{H}[t] \mathbf{h}_{i, i, j}[t]\right|^{2} \\
& \leq P_{\text {peak }}\left|\mathbf{h}_{i, i, j}[t]\right|^{2} \triangleq \mu_{i, j}^{\max }[t]
\end{aligned}
$$

where the upper bound is derived using $\sum_{j} \mathbf{w}_{i, j}^{H}[t] \mathbf{w}_{i, j}[t] \leq$ $P_{\text {peak }} \forall i$ and the fact that for two vectors $\mathbf{x}$ and $\mathbf{y}$, the quantity $|\mathbf{x y}|^{2}$ is maximized when $\mathbf{x}=\mathbf{y}^{H}$. Using the bounds of 29) and noting that all the quantities are bounded, we have,

$$
A_{i, j}^{2}[t]+\mu_{i, j}^{2}[t] \leq\left(A_{i, j}^{\max }[t]\right)^{2}+\left(\mu_{i, j}^{\max }[t]\right)^{2} .
$$

From (28), (31) and substituting the expressions for $A_{i, j}[t]$ and $\mu_{i, j}[t]$, we obtain,

$$
\begin{aligned}
& \Delta(\mathbf{Q}[t]) \leq \sum_{i, j} \mathbb{E}\left[\left(A_{i, j}^{\max }[t]\right)^{2}+\left(\mu_{i, j}^{\max }[t]\right)^{2}\right] \\
& +\sum_{i, j} Q_{i, j}[t] \lambda_{i, j}-\sum_{i, j} \mathbb{E}\left[Q _ { i , j } [ t ] \left(\left|\mathbf{w}_{i, j}^{H}[t] \mathbf{h}_{i, i, j}[t]\right|^{2}\right.\right. \\
& \left.\left.-\sum_{\substack{(n, k) \\
\neq(i, j)}}\left|\mathbf{w}_{n, k}^{H}[t] \mathbf{h}_{n, i, j}[t]\right|^{2}\right) \mid \mathbf{Q}(t)\right] .
\end{aligned}
$$

Adding the term $V \mathbb{E}\left[\sum_{i, j} \mathbf{w}_{i, j}^{H}[t] \mathbf{w}_{i, j}[t] \mid \mathbf{Q}[t]\right]$ to both the sides of the we obtain,

$$
\begin{gathered}
\Delta(\mathbf{Q}[t])+V \mathbb{E}\left[\sum_{i, j} \mathbf{w}_{i, j}^{H}[t] \mathbf{w}_{i, j}[t] \mid \mathbf{Q}[t]\right] \leq C_{1} \\
+\sum_{i, j} Q_{i, j}[t] \lambda_{i, j}-\sum_{i, j} \mathbb{E}\left[Q _ { i , j } [ t ] \left(\left|\mathbf{w}_{i, j}^{H}[t] \mathbf{h}_{i, i, j}[t]\right|^{2}\right.\right. \\
\left.-\sum_{\substack{(n, k) \\
\neq(i, j)}}\left|\mathbf{w}_{n, k}^{H}[t] \mathbf{h}_{n, i, j}[t]\right|^{2}\right) \\
\left.-V \mathbf{w}_{i, j}^{H}[t] \mathbf{w}_{i, j}[t] \mid \mathbf{Q}[t]\right] .
\end{gathered}
$$

\section{A. Part II : Proof of Proposition 3}

From (33), for the DBF policy we have,

$$
\begin{aligned}
& \Delta(\mathbf{Q}[t])+V \mathbb{E}\left[\sum_{i, j}\left(\mathbf{w}_{i, j}^{\mathrm{opt}}\right)^{H}[t] \mathbf{w}_{i, j}^{\mathrm{opt}}[t] \mid \mathbf{Q}[t]\right] \leq C_{1} \\
& +\sum_{i, j} Q_{i, j}[t] \lambda_{i, j}-\sum_{i, j} \mathbb{E}\left[Q _ { i , j } [ t ] \left(\left.\left(\mid \mathbf{w}_{i, j}^{\mathrm{opt}}[t]\right)^{H} \mathbf{h}_{i, i, j}[t]\right|^{2}\right.\right. \\
& \left.-\sum_{\substack{(n, k) \\
\neq(i, j)}}\left|\left(\mathbf{w}_{n, k}^{\mathrm{opt}}[t]\right)^{H} \mathbf{h}_{n, i, j}[t]\right|^{2}\right) \\
& \left.-V\left(\mathbf{w}_{i, j}^{\mathrm{opt}}[t]\right)^{H} \mathbf{w}_{i, j}^{\mathrm{opt}}[t] \mid \mathbf{Q}[t]\right] \\
& \stackrel{(a)}{\leq} \\
& \leq C_{1} \\
& +\sum_{i, j} Q_{i, j}[t] \lambda_{i, j}-\sum_{i, j} \mathbb{E}\left[\left.Q_{i, j}[t]\left(\mid \mathbf{w}_{i, j}^{\mathrm{TS}}[t]\right)^{H} \mathbf{h}_{i, i, j}[t]\right|^{2}\right. \\
& \left.-\sum_{\substack{(n, k) \\
\neq(i, j)}}\left|\left(\mathbf{w}_{n, k}^{\mathrm{TS}}[t]\right)^{H} \mathbf{h}_{n, i, j}[t]\right|^{2}\right) \\
& \left.-V\left(\mathbf{w}_{i, j}^{\mathrm{TS}}[t]\right)^{H} \mathbf{w}_{i, j}^{\mathrm{TS}}[t] \mid \mathbf{Q}[t]\right]
\end{aligned}
$$

where the beamforming vector $\mathbf{w}_{i, j}^{\mathrm{TS}}$ is the one implemented with any stationary randomized policy. Inequality $(a)$ follows due to the following reason. Recall that the DBF algorithm is implemented to maximize the RHS of the bound in (34). Therefore, replacing with any other policy should yield the inequality of $(a)$.

In particular we will replace by a stationary randomized policy which satisfies the following conditions.

$$
\begin{aligned}
& \mathbb{E}\left[\left|\left(\mathbf{w}^{\mathrm{TS}}{ }_{i, j}[t]\right)^{H} \mathbf{h}_{i, i, j}[t]\right|^{2}-\right. \\
& \left.\sum_{\substack{(n, k) \\
\neq(i, j)}}\left|\left(\mathbf{w}_{n, k}^{\mathrm{TS}}[t]\right)^{H} \mathbf{h}_{n, i, j}[t]\right|^{2} \mid \mathbf{Q}(t)\right] \geq \lambda_{i, j}+\epsilon, \forall i, j \\
& \mathbb{E}\left[\sum_{i, j}\left(\mathbf{w}^{\mathrm{TS}}{ }_{i, j}[t]\right)^{H} \mathbf{w}_{i, j}[t] \mid \mathbf{Q}(t)\right]=P_{\text {inf }}(\epsilon)
\end{aligned}
$$

for some $\epsilon>0$. The existance of such a policy is proved in [14] for any $\lambda_{i, j}$ lying strictly inside the achievable QoS region. Using (36) and (37) in (35) yields,

$$
\begin{aligned}
& \Delta(\mathbf{Q}(t))+V \mathbb{E}\left[\sum_{i, j}\left(\mathbf{w}_{i, j}^{\mathrm{opt}}[t]\right)^{H} \mathbf{w}_{i, j}^{\mathrm{opt}}[t] \mid \mathbf{Q}[t]\right] \leq C_{1} \\
& +\sum_{i, j} Q_{i, j}(t) \lambda_{i, j}-\sum_{i, j} Q_{i, j}(t)\left(\lambda_{i, j}+\epsilon\right)-V P_{\mathrm{inf}}(\epsilon) \\
& \quad=N K B_{1}-\epsilon \sum_{i, j} Q_{i, j}(t)-V P_{\text {inf }}(\epsilon)
\end{aligned}
$$

From (38) and from the result of Theorem 2, [14] (Lyapunov Optimization), we can conclude that,

$$
\lim _{T \rightarrow \infty} \frac{1}{T} \sum_{t=0}^{T-1} \sum_{i, j} \mathbf{E}\left[Q_{i, j}^{\mathrm{opt}}[t]\right] \leq \frac{C_{1}+V N K P_{\mathrm{peak}}}{\epsilon}
$$

and

$$
\lim _{T \rightarrow \infty} \frac{1}{T} \sum_{t=0}^{T-1} \sum_{i} \mathbf{E}\left[P_{i}^{\mathrm{opt}}[t]\right] \leq P_{\mathrm{inf}}+\frac{C_{1}}{V}
$$




\section{REFERENCES}

[1] GreenTouch Consortium, "2010-2011 Annual report," whitepaper.

[2] T. C. Group and G. e Sustainability Initiative (GeSI), "SMART 2020: Enabling the Low Carbon Economy in the Information Age." [Online]. Available: http://www.smart2020.org/

[3] G. Fettweis and E. Zimmermann, "ICT Energy Consumption - Trends and Challenges," in The 11th International Symposium on Wireless Personal Multimedia Communications (WPMC'08), 2008.

[4] E. Belmega and S. Lasaulce, "Energy-Efficient Precoding for MultipleAntenna Terminals," IEEE Trans. Signal Process., vol. 59, no. 1, pp. 329-340, Jan. 2011.

[5] S. Cui, A. Goldsmith, and A. Bahai, "Energy-efficiency of MIMO and Cooperative MIMO Techniques in Sensor Networks," IEEE J. Sel. Areas Commun., vol. 22, no. 6, pp. 1089-1098, Aug. 2004.

[6] H. Kim, C.-B. Chae, G. de Veciana, and R. Heath, "A Cross-Layer Approach to Energy Efficiency for Adaptive MIMO Systems Exploiting Spare Capacity," IEEE Trans. Wireless Commun., vol. 8, no. 8, pp. 42644275, Aug. 2009.

[7] H. S. Kim and B. Daneshrad, "Energy-Constrained Link Adaptation for MIMO OFDM Wireless Communication Systems," IEEE Trans. Wireless Commun., vol. 9, no. 9, pp. 2820-2832, Sep. 2010.

[8] Z. Chong and E. Jorswieck, "Energy Efficiency in Random Opportunistic Beamforming," in Proc. of IEEE 73rd Vehicular Technology Conference, Budapest, Hungary (VTC Spring), 2011.

[9] —_, "Energy-Efficient Power Control for MIMO Time-Varying Channels," in IEEE Online Conference on Green Communications (GreenCom), Sep. 2011, pp. 92-97.

[10] E. Uysal-Biyikoglu, B. Prabhakar, and A. El Gamal, "Energy-Efficient Packet Transmission over a Wireless Link," IEEE/ACM Trans. Netw., vol. 10, no. 4, pp. 487-499, Aug. 2002.

[11] R. Berry and R. Gallager, "Communication over fading channels with delay constraints," IEEE Trans. Inf. Theory, vol. 48, no. 5, pp. 11351149, May 2002.

[12] M. J. N. L. Georgiadis and L. Tassiulas, Resource Allocation and CrossLayer Control in Wireless Networks. Now Publishers, 2006.

[13] M. Neely and R. Urgaonkar, "Opportunism, Backpressure, and Stochastic Optimization with the Wireless Broadcast Advantage," in 42nd Asilomar Conference on Signals, Systems and Computers (ASILOMAR'08), Oct. 2008, pp. 2152-2158.

[14] M. Neely, "Energy Optimal Control for Time-Varying Wireless Networks," IEEE Trans. Inf. Theory, vol. 52, no. 7, pp. 2915-2934, Jul. 2006.

[15] M. Gastpar, "On Capacity Under Receive and Spatial Spectrum-Sharing Constraints," IEEE Trans. Inf. Theory, vol. 53, no. 2, pp. 471-487, Feb. 2007.

[16] R. Zhang, Y. Liang, and S. Cui, "Dynamic Resource Allocation in Cognitive Radio Networks ," IEEE Signal Process. Mag., vol. 5, no. 27, pp. 102-114, May 2010.

[17] R. Zhang, "On Peak versus Average Interference Power Constraints for Protecting Primary Users in Cognitive Radio Networks," IEEE Trans. Signal Process., vol. 8, no. 4, pp. 2112-2120, Apr. 2009.

[18] E. Nekouei, H. Inaltekin, and S. Dey, "Throughput Scaling in Cognitive Multiple-Access with Average Power and Interference Constraints," IEEE Trans. Signal Process., vol. 60, no. 2, pp. 927-946, Feb. 2012.

[19] M. Neely, Stochastic Network Optimization with Application to Communication and Queueing Systems. Morgan \& Claypool, 2010.

[20] J. F. Sturm, "Using SEDUMI 1.02, a Matlab Toolbox for Optimizations Over Symmetric Cones," Optimization Meth. and Soft, vol. 11-12, May 1999.

[21] G. Pataki, "On the Rank of Extreme Matrices in Semidefinite Programs and the Multiplicity of Optimal Eigenvalue," Math. Oper. Res., vol. 23, no. 2, pp. 339-358, 1998. 